\title{
Computational modeling and analysis of prominent T-cell epitopes for assisting in designing vaccine of ZIKA virus
}

\author{
Garima Yadav $\$$, Richa Rao\$, Utkarsh Raj $\$$, Pritish Kumar Varadwaj* \\ Department of Bioinformatics \& Applied Sciences, Indian Institute of Information Technology-Allahabad, Allahabad, Uttar Pradesh, India.
}

\section{ARTICLE INFO}

Article history:

Received on: 07/04/2017

Accepted on: 09/05/2017

Available online: 30/08/2017

Key words:

Vaccine, Zika virus, T-cell

epitope, MHC class II.

\begin{abstract}
The Zika virus disease or Zika fever, regularly shows no or just mellow side effects, like an exceptionally gentle type of dengue fever. It spread eastwards from 2007-16 over the Pacific Ocean to the Americas, whereas in 2015 to 2016, Zika virus scourge achieved epidemic levels. In this study, the antigenic epitopes of Zika virus (ZIKV) were predicted and modeled. The highest binding scorers among the predicted ones and their correlating MHC class II alleles were further subjected to binding simulation studies. Immunoinformatics tools were applied to analyze the viral antigenic proteins that may be helpful in designing vaccine for ZIKV. The promiscuous epitopes of MHC class II were predicted from the viral proteins using ProPred, an immunoinformatics tool. The chosen epitopes and MHC alleles were modeled molecularly using PEP-FOLD3 and CPH model 3.2 servers respectively. The viral glycoprotein having epitope/peptide YRIMLSVHG bound to DRB1*01:01 MHC class II allele demonstrated the most noteworthy binding score. The anticipated peptide has high possibility of inducing $\mathrm{T}$ cell mediated immune response and it might be helpful in designing vaccines based on epitopes after continued future trials.
\end{abstract}

\section{INTRODUCTION}

Zika virus (ZIKV) is an arbovirus of the genus Flavivirus and the family Flaviviridae (Lanciotti et al., 2008). It is a positive-sense single-stranded RNA which incorporates a few other mosquito-borne infections of clinical significance such as dengue virus (DNV), yellow fever virus (YFV), and West Nile virus (WNV) (Lanciotti et al., 2008). The other individual from its clade, the Spondweni virus, is its nearest relative (Lanciotti et al., 2008; Kuno and Chang, 2007). The genome of Zika virus contains 10,794 nucleotides which encode 3,419 amino acids Kuno and Chang, 2007). The Zika virus is similar to other flaviviruses, that is, it is made out of 2 non-coding regions ( $3^{\prime}$ and 5') by which a polyprotein is encoded and a single open reading frame (ORF) is flanked. This encoded polyprotein gets cleaved

Corresponding Author

Email: pritish@iiita.ac.in

${ }^{\$}$ These authors having equal contribution. into a triad of structural proteins, viz. precursor of membrane (prM), envelope (E) and capsid (C); and seven non-structural proteins (Kuno and Chang, 2007). The day-time active mosquitoes, predominantly of the Aedes (Stegomyia) genus, such as A. aegypti and A. albopictus, transmit the ZIKV (Malone et al., 2016). Aedes mosquitoes are generally circulated all around the globe, and most of the mosquito species inhabit warm subtropical and tropical local natural surroundings (Kraemer et al., 2015). A. albopictus does not yet give off an impression of being a noteworthy vector of ZIKV. But we can't ignore its role in the Gabon flare-up of 2007, it's widespread all through the US and absence of ZIKV when this particular species Aedes sp. was limited (Kraemer et al., 2015).

Inspite of various researches carried for treating ZIKV infection, there is no proper standard treatment available till date for curing this deadly infection. The administration is steady and incorporates rest, liquids, antipyretics, and analgesics. Headache medicines and other non-steroidal anti-inflammatory medications should not be taken until dengue is treated completely as this possesses the danger of hemorrhage (Kuno and Chang, 2007). 
So vaccine development against the ZIKV is very essential. The most antigenic part of a virus is its surface or envelope proteins which are often considered as a great contender for inoculation. The adaptive immune response primarily targets the envelope protein which mediates the viral entry which makes them crucial for vaccine development (Cerdeño-Tárraga et al., 2003; Trent and Qureshi, 1971). Over the past few years, immunologists have discovered the epitopes which can be identified by $\mathrm{T}$ cells and $\mathrm{B}$ cells are different and this has resulted in designing of more potent candidates for vaccine. Intercellular foreign antigenic peptides recognition majorly involves Major histocompatibility complex (MHC) and hence it takes part in developing both cell-mediated and humoral immune responses. Antigen-presenting cells (APCs) contain T cells on their surface which recognize antigenic fragments only when the exposed surface MHC molecules of all vertebrate cells are combined with them (Shekhar et al., 2012; Mohabatkar and Mohammadzadegan, 2007)

The heterodimeric glycoproteins, MHC molecules, induce the activation of $\mathrm{T}$ cell by presenting a varied peptide set on the cell surrounding surface (Viret and Janeway, 1999; Tambunan and Parikesit, 2011). In a population with various alleles, the binding regions of an allele may not trigger the immune response due to high polymorphism of MHC molecules. So it's more necessary to identify viral peptides that are promiscuous and possess binding capacity with multiple MHC alleles (Tambunan and Parikesit, 2011).

Epitope or peptide-based vaccines are more specific, worthwhile, easily producible, safer and more time efficient than the conventional vaccines. The epitope or peptide-based vaccines can deliver high immunogen dosage at lower cost (Von Hoff et al., 2005; Tang et al., 2012). In order to design a potent synthetic peptide vaccine candidate, In Silico modeling techniques and immunoinformatics approaches have been applied including the use of bioinformatics software and tools which are based on various machine learning programs and other statistical approaches. The active candidate for vaccine must possess antigenicity and it must be responsible for pathogenicity (De Groot et al., 2002; Verma et al., 2011)

\section{METHODS AND MATERIALS}

\section{Retrieving Zika virus envelope glycoprotein sequence and Protein antigenicity determination}

Zika virus envelope (outer membrane) glycoprotein sequences were extracted in FASTA format from UniProtKB (http://www.uniprot.org/) (Gaunt et al., 2001). Theoretical isoelectric point (pI), molecular weight and amino acid compositions were computed by using the Expasy's ProtParam server for all the 40 glycoprotein sequences (Chandra et al., 2010). These sequences were then investigated with VaxiJen (http://www.ddg-pharmfac.net/vaxijen/VaxiJen/VaxiJen.html) with default parameters to discover its antigenicity, to predict the protective antigen as a vaccine (Doytchinova and Flower, 2007).
Amongst all the antigenic proteins, the one having the highest antigenicity score was chosen to carry out further studies.

\section{T-cell epitope-bound class II HLA expectation and identification}

To identify the promiscuous $\mathrm{T}$ cell epitopes various distinctive prognosis procedures have been utilized. Epitopes are known as "promiscuous" when more than one MHC allele and more than one $\mathrm{T}$ cell clone can identify different $\mathrm{T}$ cell epitopes.

For the prediction of linear peptides as $\mathrm{CD} 4+\mathrm{T}$-cell epitopes for ZIKV envelope glycoprotein (GP) we picked abundant human leukocyte antigen class II alleles in humans (DRB1*07:01, DRB1*01:01， DRB1*04:01， DRB1*08:02, DRB1*03:01, DRB1*11:01, DRB1*15:01, DRB1*13:02 $)^{[19]}$ and used ProPred (http://www.imtech.res.in/raghava/propred/), a graphical web-based tool which predicts those regions in an antigenic sequence which binds to MHC class-II. ProPred locates promiscuous binding sites in assistance with quantitative matrices. These binding regions are used for selecting candidates for vaccines (Gowthaman and Agrewala, 2007; Sturniolo et al., 1999). The predicted epitopes were further validated by using PREDIVAC (http://predivac.biosci.uq.edu.au/cgi-bin/binding.py), which predicts peptide binding of HLA class II on the basis of specificity-determining residue (SDR) concept covering 95\% allelic variants of MHC class II (DR locus) and IEDB Analysis Resource (http://www.iedb.org/) which involves various MHC Class-II epitope prediction method, involving a consensus approximation combining $\mathrm{NN}$-align, combinatorial library methods and SMM-align (Oyarzun et al., 2013). In this study, we have used the SMM prediction method.

\section{Modeling favorable epitopes and MHC II alleles and their computational validations}

The GP epitopes of ZIKV were modeled at PEPFOLD3 (http://bioserv.rpbs.univparisdiderot.fr/services/PEP-FOLD3/) for each MHC allele. In the Protein Data Bank (PDB) the 3-D structures of MHC alleles were inaccessible, so in order to secure their 3-D coordinate's files CPH model 3.2 server which uses their respective structural templates, was used. Afterwards, numerous online servers were used for verification and structural analysis of MHC alleles' structure. The servers being used are Errat, ProQ, ProSA, RAMPAGE, etc. Errat (http://services.mbi.ucla.edu/ERRAT/) is an algorithm for verification of protein structure which is used specifically for analyzing non-bonded interaction statistics between various atom types (Colovos and Yeates, 1993). ProQ (http://www.sbc.su.se/ bjornw/ProQ/ProQ.cgi) is a protein quality prediction tool that predicts the nature of a protein model on the basis of MaxSub and LG score, the two quality measures (Wallner and Elofsson, 2003). ProSA (https://prosa.services.came.sbg.ac.at/prosa.php) is a web-based tool which analyzes the structure of a protein (Wiederstein and Sippl, 2007). It determines the model quality both local and overall (Z-score) and for a particular protein structure it calculates an 
overall quality score. RAMPAGE (http://mordred.bioc.cam.ac.uk/ rapper/rampage.php) is a web tool for assessing protein model on the basis of Ramachandran plot (Lovell et al., 2002).

\section{Docking prediction of epitopes and alleles}

After modeling the structure, chosen peptides and alleles were docked using rigid docking method with the assistance of PatchDock followed by refinement through FireDock to decide the values of binding score.

\section{Epitope-HLA allele molecular dynamic simulation}

The molecular dynamic simulation was performed by utilizing Desmond (Klepeis et al., 2009; Raj et al., 2015). Initial co-ordinates were obtained from the best peptide-allele complex for dynamics simulation process. The system building process was carried out by adding Simple Point Charge water model. Then the system was neutralized with the addition of counter ions followed by the system minimization step using Broyden-Fletcher-Gold farb-Shanno LBFGS algorithms. The whole system was further subjected for MD simulation studies for $20 \mathrm{~ns}$ at $300 \mathrm{~K} \& 1.0325$ bar pressure and the RMSD was recorded to check the stability of the above said complex (Raj et al., 2016; Kamal et al., 2016).

\section{RESULTS AND DISCUSSION}

\section{Recovery and prediction of antigenic Protein}

Sequence analysis of envelope glycoprotein segregated from Zika virus was done on the basis of the structure. Fasta format of an aggregate of 40 polyproteins was recovered from UniProtKB. The physiochemical properties for all these 40 poyproteins hasve been summarized in Table 1. The protein having the highest immunogenicity was predicted using VaxiJen. The envelope protein, UniProtKB ID Q91KX7, was predicted with highest antigenicity with an aggregate score of 0.6269 at a threshold value of 0.4 . This prediction corresponds to a previous observation where immunogenicity of the envelope glycoprotein was found (Roehrig et al., 1983).

\section{Prediction and analysis of MHC Class II binding peptides}

Based on the scores generated by the Propred (Table 2), peptides showing maximum binding with the same alleles of MHC II were taken.

Table 1: Physiochemical Properties of proteins.

\begin{tabular}{|c|c|c|c|c|}
\hline Protein Designation & Accession No. & Size aa & Expected MW kDA & pI \\
\hline Envelope protein & Q91KX7 & 276 & 30299.52 & 6.23 \\
\hline Envelope protein & A0A060H177 & 504 & 54380.19 & 6.51 \\
\hline Envelope protein & W8QIQ6 & 251 & 27562.29 & 5.82 \\
\hline Envelope protein & W8QIN8 & 251 & 27504.21 & 5.82 \\
\hline Envelope protein & A0A0N7HJ59 & 103 & 10840.53 & 8.87 \\
\hline Envelope protein & A0A0H4A724 & 110 & 11753.47 & 8.84 \\
\hline Envelope protein & W8R1P6 & 251 & 27532.20 & 5.82 \\
\hline Envelope protein & W8R1M7 & 251 & 27500.14 & 5.93 \\
\hline Envelope protein & A0A0N9ZT66 & 103 & 10840.53 & 8.87 \\
\hline Envelope protein & A0A0H4AEI9 & 110 & 11753.47 & 8.84 \\
\hline Envelope protein & W8Q7L0 & 251 & 27504.21 & 5.82 \\
\hline Envelope protein & W8Q6P9 & 251 & 27532.20 & 5.82 \\
\hline Envelope protein & W8QIP2 & 251 & 27544.27 & 5.82 \\
\hline Envelope protein & W8QFD0 & 251 & 27474.27 & 6.04 \\
\hline Envelope protein & W8Q6N7 & 251 & 27586.35 & 5.82 \\
\hline Envelope protein & W8R1N8 & 251 & 27504.21 & 5.82 \\
\hline Envelope protein & W8R1M9 & 251 & 27504.21 & 5.82 \\
\hline Envelope protein & W8Q7L5 & 251 & 27474.27 & 6.04 \\
\hline Envelope protein & W8QFD5 & 251 & 27522.22 & 5.93 \\
\hline Envelope protein & W8QFB7 & 251 & 27558.30 & 5.93 \\
\hline Envelope protein & W8QIM9 & 251 & 27603.34 & 5.94 \\
\hline Envelope protein & W8PAE0 & 280 & 30572.76 & 6.14 \\
\hline Envelope protein & W8QFC5 & 251 & 27512.27 & 5.82 \\
\hline Envelope protein & W8QIQ1 & 251 & 27405.26 & 5.71 \\
\hline Envelope protein & W8R1P2 & 251 & 27532.20 & 5.82 \\
\hline Envelope protein & W8Q6N2 & 251 & 27589.32 & 5.94 \\
\hline Envelope protein & W8QFD9 & 251 & 27546.29 & 5.82 \\
\hline Envelope protein & W8Q7L9 & 251 & 27535.22 & 5.71 \\
\hline Envelope protein & W8QFC1 & 251 & 27523.25 & 5.87 \\
\hline Envelope protein & W8Q6P1 & 251 & 27516.26 & 5.82 \\
\hline Envelope protein & W8Q7J5 & 251 & 27589.32 & 5.94 \\
\hline Envelope protein & W8Q6P5 & 251 & 27562.29 & 5.82 \\
\hline Envelope protein & A0A0P0A2N5 & 103 & 10840.48 & 8.53 \\
\hline Envelope protein & W8QIN4 & 251 & 27599.36 & 5.94 \\
\hline Envelope protein & W8QFB3 & 251 & 27599.35 & 5.87 \\
\hline Envelope protein & W8Q6N5 & 251 & 27504.21 & 5.82 \\
\hline Envelope protein & W8Q7K0 & 251 & 27504.21 & 5.82 \\
\hline Envelope protein & A0A142JYW4 & 120 & 12983.62 & 6.98 \\
\hline Envelope protein & W8QIP7 & 245 & 26804.44 & 5.75 \\
\hline Envelope protein & W8Q7K5 & 251 & 27523.25 & 5.87 \\
\hline
\end{tabular}


Table 2: Scores generated by Propred.

\begin{tabular}{clll}
\hline Position & Epitope & \multicolumn{1}{c}{ Allele } & \multicolumn{1}{c}{ Prediction } \\
\hline \multirow{2}{*}{73} & \multirow{2}{*}{ YRIMLSVHG } & HLA- DRB1*01:01 & ProPred, IEDB, PREDIVAC \\
& & HLA- DRB1*11:01 & ProPred, IEDB, PREDIVAC \\
& & HLA- DRB1*13:02 & ProPred, IEDB, PREDIVAC \\
\hline \multirow{2}{*}{228} & \multirow{2}{*}{ LRLEGVSYS } & HLA- DRB1*03:01 & ProPred, IEDB, PREDIVAC \\
& & HLA- DRB1*04:01 & ProPred, IEDB, PREDIVAC \\
\hline 110 & LGGFGSLGL & HLA- DRB1*15:01 & ProPred, IEDB, PREDIVAC \\
\hline
\end{tabular}

Program: ERRAT2

File:/var/www/SAVES/Jobs/7614043//errat.pdb

Chain\#:1

Overall quality factor ${ }^{\star *}: 75.956$

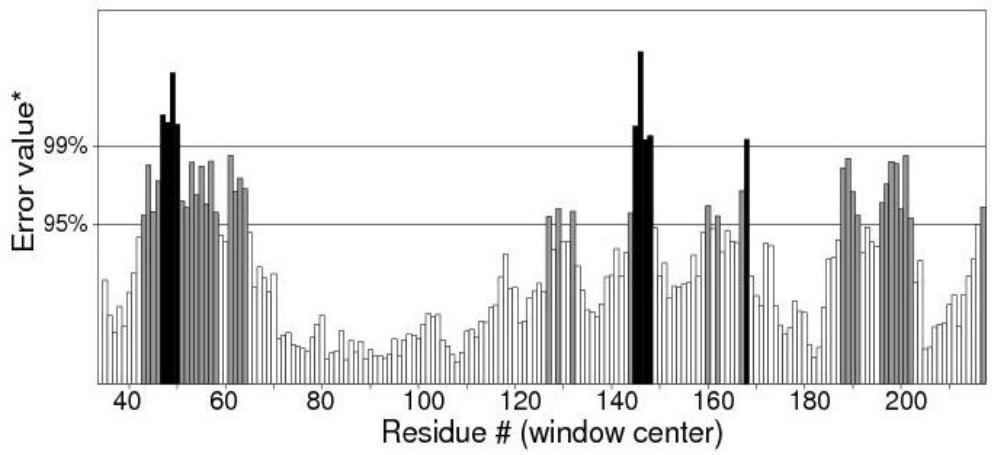

Fig. 1: The graphical representation of ERRAT result of DRB $1 * 01: 01$ allele. On the error axis, two lines are drawn to show the certainty of which it is conceivable to reject regions that surpass that error values.

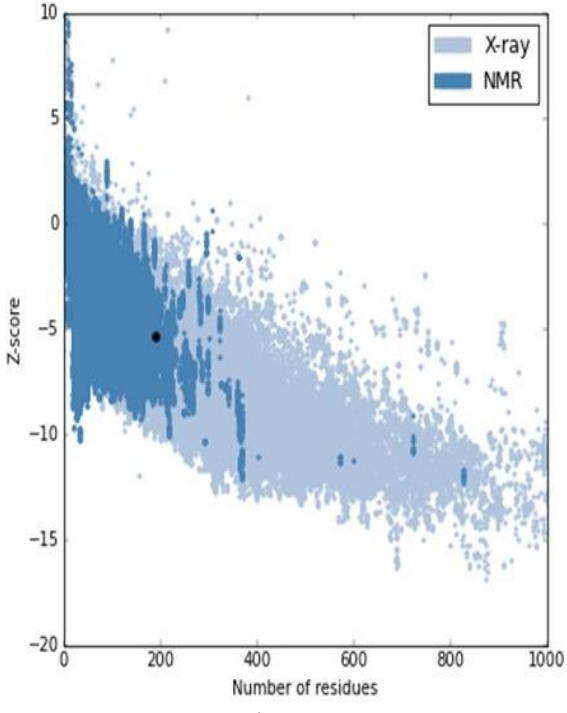

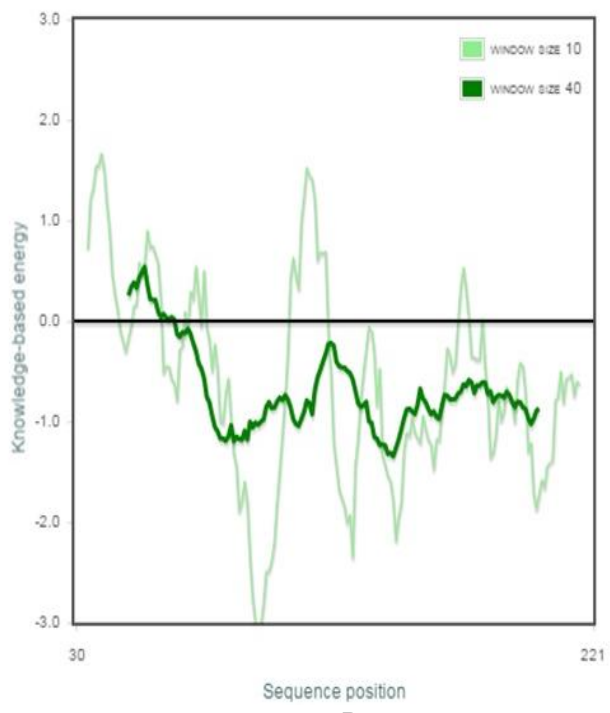

Fig. 2 ProSA result. (a) Z-score plot of DRB1*01:01 allele. (b) Graphical plot residue scores of DRB1*01:01 allele structure.

\section{Target peptides and MHC II allele modeling and validation}

PEP-FOLD3 and CPH model server were used to obtain

3-D coordinate file of favored peptides and allele respectively. The structures of DRB1*01:01, DRB1*15:01 and DRB1*04:01 alleles were validated through Errat, ProSA, RAMPAGE and ProQ. Errat was used to determine the general quality factor of an allele. It was 75.956 for DRB1*01:01(Fig. 1), 82.418 for DRB1*15:01 and 75.138 for DRB1*04:01. Quality predicted by ProQ was 'fairly good' for all the three alleles,
DRB1*01:01, DRB1*15:01 and DRB1*04:01, with LG scores of $1.935,2.125,2.241$, and MaxSub scores of $0.162,0.175,0.172$ respectively. Utilizing ProSA, the computed Z-score of alleles DRB1*01:01, DRB1*15:01 and DRB1*04:01 was - 5.39 (Fig. 2.a \& b), -5.18, -5.34 respectively and it resulted in considerably good model. Using RAMPAGE analysis, the favored region amino acid residues number was 179 (94.2\%), 178(94.2\%), 179(95.7\%) for DRB1*01:01 (Fig. 3), DRB1*15:01 and DRB1*04:01 alleles. 


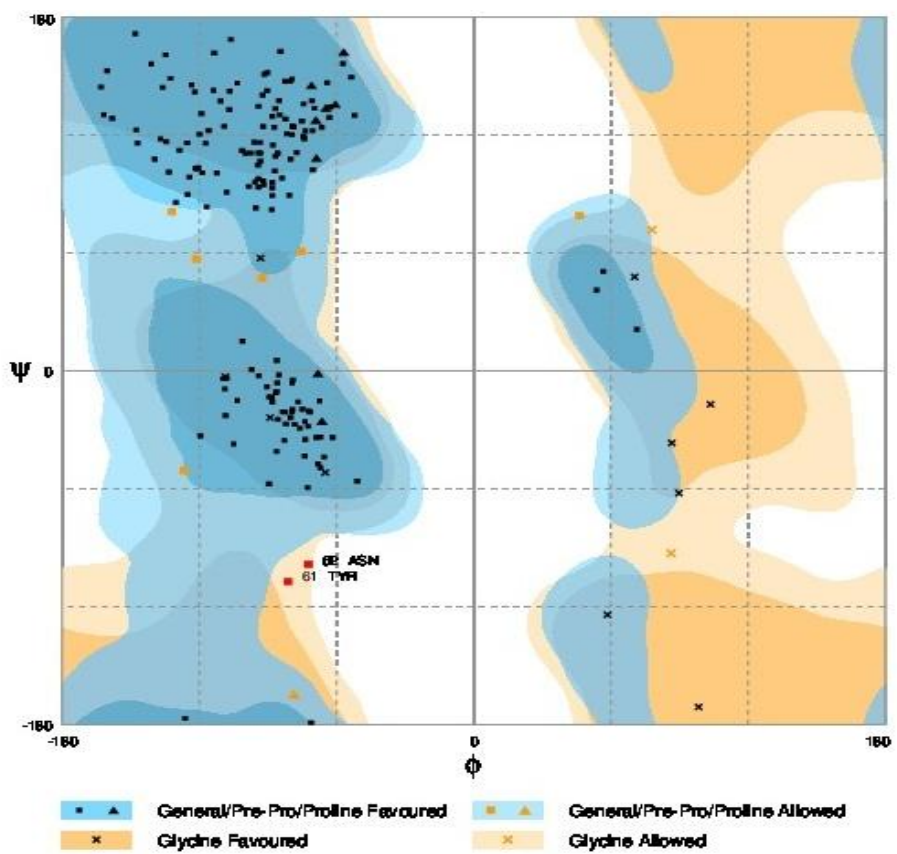

Fig.3 RAMPAGE demonstrating the residues in favored, allowed and outlier regions of DRB1*01:01 allele.

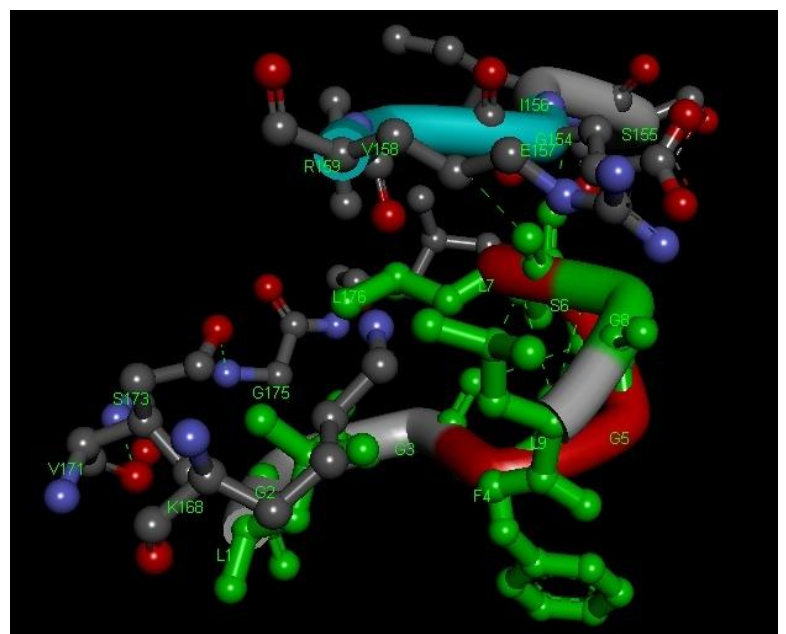

Fig.4 Docked epitope YRIMLSVHG-DRB 1*01:01 allele complex by PatchDock and FireDock portraying the detailed position of amino acids alongside the arrangement of $\mathrm{H}$-bonds.

Table 3: Docking and post docking results of epitopes with alleles.

\begin{tabular}{ccccccccc}
\hline Allele & Score & Area & Transformation & GE & Vdw Attractive & Vdw Repulsive & ACE & HB \\
\hline DRB $1 * 01: 01$ & 4852 & 530.80 & -3.0040 .542 .7593 .199 .12 & 2.76 & -57.31 & -17.02 & 11.87 & -13.46 \\
DRB $1 * 4: 01$ & 5162 & 563.20 & $-1.501 .012 .2387 .264 .91-14.51$ & -49.87 & -20.35 & -2.40 & -12.84 & -2.13 \\
DRB $1 * 15: 01$ & 6014 & 710.30 & $1.230 .02-0.2319 .6128 .87113 .67$ & -51.06 & -24.06 & 12.26 & -12.94 & -0.78 \\
\hline
\end{tabular}

\section{Docking score determination by PatchDock and refinement of docking result by FireDock}

Docking score investigations of epitopes YRIMLSVHG, LRLEGVSYS, LGGFGSLGL with DRB1*01:01, DRB1*04:01 and DRB1*15:01 alleles framed stable HLA-peptide complexes with the binding score of 4852, 5162 and 6032 respectively. Refinement through FireDock gave the global binding energy of $57.31,-49.87$ and -51.06 respectively (Table 3 ).
Further we determined the contribution of the hydrogen bonds to the global binding energy utilizing FireDock, it was found to be $-2.40,-2.13$ and -0.78 respectively.

In this way, the epitope YRIMLSVHG with DRB1* 01:01 allele demonstrates the greatest chances of efficient binding as H-bonds may form. Hydrogen bonds are present on YRIMLSVHG-DRB1*01:01 complex at position S6, S155, G8, and E157 (Fig.4). 


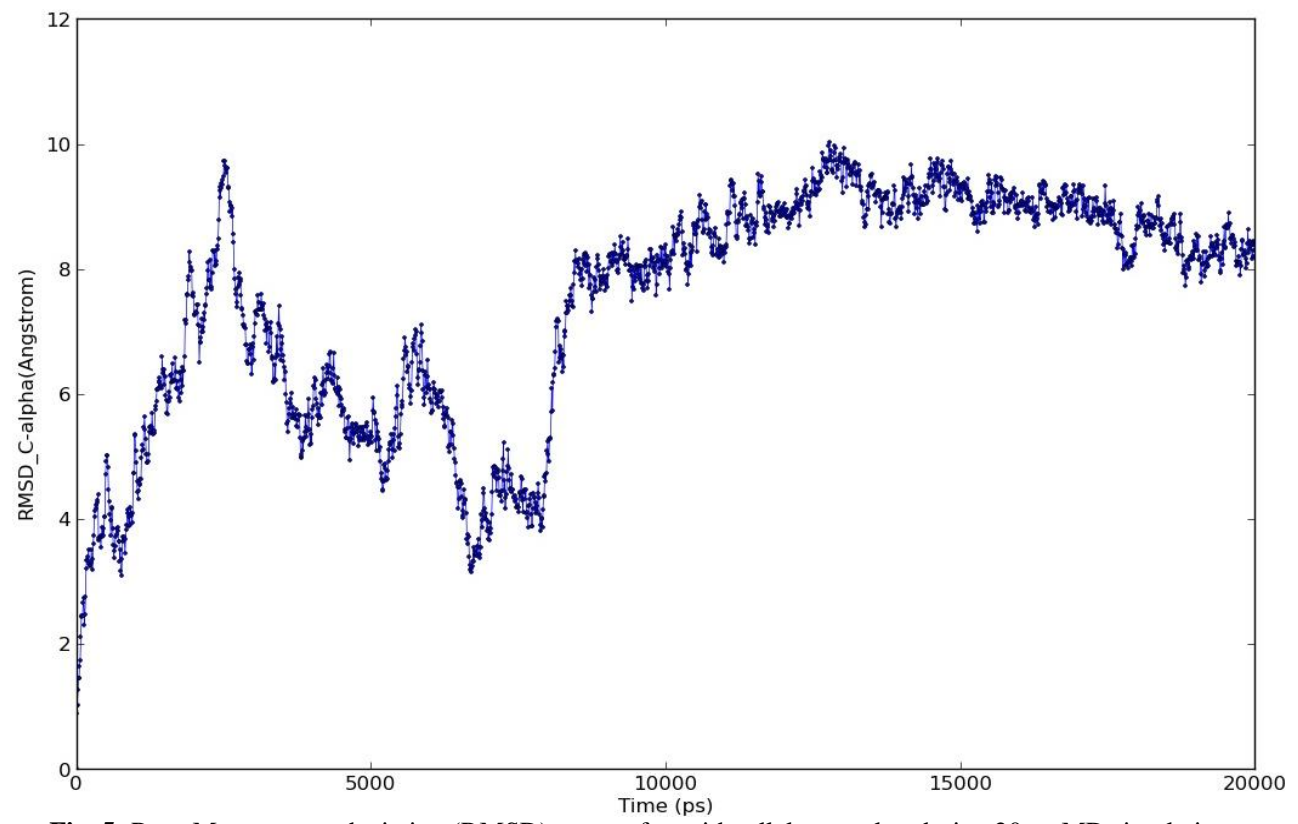

Fig. 5: Root Mean square deviation (RMSD) curve of peptide-allele complex during $20 \mathrm{~ns}$ MD simulation.

\section{Allele-peptide complex analysis using molecular dynamics simulation}

The best allele and peptide complex obtained through FireDock were subjected to molecular dynamics simulations. Peptide YRIMLSVHG-DRB1*01:01 allele complex at RMSD value of $9.8 \AA$ shows the highest peak (Fig. 5). The mean RMSD obtained during MD trajectory of 20 ns was $7.438 \AA$ with a standard deviation of $1.878 \AA$. As displayed in the graph, fluctuations occur mainly during initial phase up to $10 \mathrm{~ns}$ after that the graph shows consistent behavior which in turn reveals the stability of the complex during 10-20 ns simulation time.

\section{CONCLUSION}

The predicted epitopes from the genome/proteome sequences of the pathogens would greatly reduce the time as well as cost and be useful for experimental planning in the development of epitope vaccines. The predicted epitopes may be used for safe vaccine development against meningococcal diseases. The pathogenic activity of Zika Virus makes it difficult to develop novel vaccines. Vaccine development requires in-vivo and in-vitro experiments which are time-consuming. Virus growth and peptides' segregation on a vast cluster of tests is additionally an enormous what's more, legitimate limitation. The present study consolidates immunoinformatics approach for diminishing the time lost in the long array of experiments to maintain a strategic distance from hit and trial sets. A glycoprotein having 276 amino acids has the probability of holding an expansive number of epitopes. So out of many proteins, 276 amino acids long glycoprotein was taken to predict epitopes for T cell bound MHC II as a vaccine candidate for ZIKA envelope glycoprotein.
Vaccine designing and development against $\mathrm{T}$ cell epitopes appears convincing because the immune response evoked by it is long lasting and it also stimulates antigenic drift in which the memory response due to antibody can be easily escaped by the antigen (Trainor et al., 2007).

$\mathrm{T}$ cell bound MHC class II epitope YRIMLSVHG was predicted to have the maximum score of $63.33 \%$ with HLADRB1*01:01 allele. Errat was used to validate the homology modeling of the allele and it was found to have score of 75.956, LG score of 1.935 and MaxSub score of 0.162 obtained using ProQ, Z-score of -5.39 obtained using ProSA and with RAMPAGE resulting in 179(94.2\%) amino acids in the favored region. Docking was done by PatchDock with score 4852 and refinement by FireDock with a global energy of -57.31 . H-bond was recognized at position S6, S155, G8, and E157. An appropriately stable allele-peptide complex was accomplished during the molecular dynamic simulation interval of 10-20 ns.

It is observed that peptide YRIMLSVHG with DRB1*01:01 may prove to be the backbone in predicting design of vaccine. The peptide can be either formulated or isolated for further in-vivo and in-vitro experiments.

Financial support and sponsorship: Nil.

Conflict of Interests: There are no conflicts of interest.

\section{REFERENCES}

Lanciotti RS, Kosoy OL, Laven JJ, Velez JO, Lambert AJ, Johnson AJ., Genetic and serologic properties of Zika virus associated with an epidemic, Yap State, Micronesia, 2007. Emerg Infect Dis. 2008; 14:1232-9.

Kuno G, Chang GJ., Full-length sequencing and genomic characterization of Bagaza, Kedougou, and Zika viruses. Arch Virol. 
2007; 152:687-96

Malone RW, Homan J, Callahan MV, Glasspool-Malone J, Damodaran L, Schneider AD, Zimler R, Talton J, Cobb RR, Ruzic I, Smith-Gagen J. Zika virus: medical countermeasure development challenges. PLoS Negl Trop Dis. 2016; 2;10(3):e0004530.

Kraemer MU, Sinka ME, Duda KA, Mylne A, Shearer FM, Brady OJ., The global compendium of Aedes aegypti and Ae. albopictus occurrence. Sci Data. 2015; 2:150035.

Fauci AS, Morens DM., Zika virus in the Americas-yet another arbovirus threat. N Engl J Med. 2016; 374:601-4.

Cerdeño-Tárraga AM, Efstratiou A, Dover LG, Holden MT, Pallen M., The complete genome sequence and analysis of Corynebacterium diphtheriae NCTC13129. Nucleic Acids Res. 2003;15; 31(22):6516-23.

Trent DW, Qureshi AA. Structural and nonstructural proteins of Saint Louis encephalitis virus. Journal of virology. 1971; 1;7(3):379-88.

Shekhar C, Dev K, Verma SK, Kumar A., In-silico: screening and modeling of CTL binding epitopes of Crimean congo hemorrhagic fever virus. Trends Bioinform 2012; 5:14-24.

Mohabatkar H, Mohammadzadegan R., Computational comparison of T-cell epitopes of GP 120 of Iranian HIV-1 with different subtypes of the virus. Pak J Biol Sci 2007; 10:4295-4298.

Viret C, Janeway CA., MHC and T cell development. Rev Immunogenet 1999; 1:91-104.

Tambunan USF, Parikesit AA., In silico design of drugs and vaccines for dengue disease. Trends Bioinform 2011; 4:1-9.

Jiao W, Yin W, Li X, Liu J., Research advancement in RV novel vaccine. Asian J Anim Vet Adv 2012; 7:1234-1244.

Von Hoff DD, Evans DB, Hruban RH., Pancreatic cancer. Jones and Bartlett, Sudbury, 2005.

Tang H, Liu XS, Fang YZ, Pan L, Zhang ZW et al., The epitopes of foot and mouth disease. Asian J Anim Vet Adv 2012; 7:12611265.

De Groot AS, Sbai H, Saint Aubin C, McMurry J, Martin W. Immuno-informatics: mining genomes for vaccine components. Immunology and Cell Biology. 2002; 1;80(3):255-69.

Verma SK, Yadav SSP, Kumar A., In silico T cell antigenic determinants from proteome of H1N2 swine influenza A virus. Online J Bioinform 2011; 12:371-378.

Gaunt MW, Sall AA, de Lamballerie X, Falconar AK, Dzhivanian TI, Gould EA. Phylogenetic relationships of flaviviruses correlate with their epidemiology, disease association and biogeography. Journal of General Virology. 2001; 1;82(8):1867-76.

Chandra S, Singh D, Singh TR. Prediction and characterization of T-cell epitopes for epitope vaccine design from outer membrane protein of Neisseria meningitidis serogroup B. Bioinformation. 2010; 1; 5(4):15561.

Doytchinova IA, Flower DR. VaxiJen: a server for prediction of protective antigens, tumour antigens and subunit vaccines. BMC Bioinformatics. 2007; 8(4).

Gowthaman U., Agrewala J. N., In silico tools for predicting peptides binding to HLA-class II molecules: more confusion than conclusion. Journal of proteome research 2007; 7(01): 154-163.
Sturniolo T, Bono E, Ding J, Raddrizzani L, Tuereci O, Sahin U,Braxenthaler M, Gallazzi F, Protti MP, Sinigaglia F, Hammer J, Generation of tissue-specific and promiscuous HLA ligand database using DNA microarrays and virtual HLA class II matrices. Nat Biotechnol. 1999; 17:555-561.

Oyarzun P, Ellis JJ, Boden M and Kobe B. PREDIVAC: CD4+ T-cell epitope prediction for vaccine design that covers $95 \%$ of HLA class II protein diversity. BMC Bioinformatics, 2013; 14:52.

Colovos C, Yeates TO. Verification of protein structures: patterns of non-bonded atomic interactions. Protein Sci. 1993; 2:15111519

Wallner B, Elofsson A. Can correct protein models be identified. Protein Sci. 2003; 12:1073-1086.

Wiederstein M, Sippl MJ., ProSA-web: interactive web service for the recognition of errors in three-dimensional structures of proteins Nucleic Acids Res. 2007; 35:407-410.

Lovell SC, Davis IW, Arendall WB III, De Bakker PIW, Word JM, Prisant MG, Richardson JS, Richardson DC. Structure validation by Calpha geometry: phi, psi and Cbeta deviation. Proteins. 2002; 50:437450 .

Klepeis JL, Lindorff-Larsen K, Dror RO, Shaw DE. Longtimescale molecular dynamics simulations of protein structure and function. Current opinion in structural biology. 2009; 19(2):120-7.

Raj U, Kumar H, Gupta S, Varadwaj PK. Novel DOT1L receptor natural inhibitors involved in mixed lineage leukemia: A virtual screening, molecular docking and dynamics simulation study. Asian Pacific Journal of Cancer Prevention. 2015; 16(9):3817-25.

Kumar Chaudhary K, Raj U, Kumar Varadwaj P, Mishra N. Novel Potent Plasmepsin-I (PMI) Inhibitors: An In-Silico Approach Current Proteomics. 2016; 1;13(3):196-205.

Raj U, Kumar H, Varadwaj PK. Molecular docking and dynamics simulation study of flavonoids as BET bromodomain inhibitors. Journal of Biomolecular Structure and Dynamics. 2016; 5:1-12.

Roehrig JT, Mathews JH, Trent DW. Identification of epitopes on the E glycoprotein of Saint Louis encephalitis virus using monoclonal antibodies. Virology. 1983; 128(1):118-26.

Trainor NB, Crill WD, Roberson JA, Chang GJ. Mutation analysis of the fusion domain region of St. Louis encephalitis virus envelope protein. Virology. 2007; 10; 360(2):398-406.

\section{How to cite this article:}

Yadav G, Rao R, Raj U, Varadwaj PK. Computational modeling and analysis of prominent T-cell epitopes for assisting in designing vaccine of ZIKA virus. J App Pharm Sci, 2017; 7 (08): 116-122. 